\title{
Helicopter rotation and smectic-isotropic coexistence of strongly attractive rods
}

\author{
Marisol Ripoll* \\ Theoretical Soft Matter and Biophysics, Institute of Complex Systems, Forschungszentrum Jülich, D-52425 Jülich, Germany
}

(Received 12 October 2010; published 29 April 2011)

\begin{abstract}
Hydrodynamic simulations of strongly attractive rodlike colloids are performed with and without shear flow. In the absence of flow, the isotropic-nematic coexistence becomes isotropic-smectic A, and the interfacial properties clearly vary with increasing attraction strength. In the presence of shear flow, a new collective rotation appears in which the director rotates in the vorticity-flow plane in a similar fashion to the movement of the rotor of a helicopter.
\end{abstract}

DOI: 10.1103/PhysRevE.83.040701

PACS number(s): 64.70.M-, 82.70.Dd, 64.60.-i, 83.50.-v

Liquid crystals in equilibrium, and in the presence of flow fields, show a very rich structural and dynamical behavior. Equilibrium phase diagrams display coexistence between isotropic (I), where rods are randomly oriented, nematic (N), where rods have a orientational preference, smectic (Sm), with both orientational and positional order, and crystalline phases, where the positional order is long ranged [1]. Attraction induced by depletion interactions has been considered in analytical calculations and experiments [2,3] of rods with large aspect ratios (rod length $L$ over rod diameter $\sigma$ ). The broadening of existing coexistence regions like I-N and N-Sm has been determined, together with the appearance of new ones as I-I, N-N, and eventually also I-Sm. Computer simulations are a powerful tool that can provide a detailed description of these systems. Numerous simulations with repulsive rods of small aspect ratios, typically from 2 to 6 , have studied phases with positional order (see, e.g., Ref. [4]). Simulations of thermotropic systems of Gay-Berne colloidal rods [5] with increasing attraction have reported the appearance of different phases as a function of the potential asymmetry. Gay-Berne forces become unrealistically thin at the ends with increasing aspect ratio. I-Sm coexistence has been obtained with such rods by keeping the two phases at different temperatures [6]. In the presence of shear flow, the phase diagram is fundamentally altered [7]. Isotropic phases tend to align with flow, and oriented phases may undergo orchestrated motions that have been characterized about two decades ago [8]. Nematic phases have shown rotations in the flow-gradient plane (similar to the blades of a mill) or with an angle tilted in the vorticity direction (similar to the paddle in a kayak). These rotations are known as tumbling and kayaking [9]. Emerging questions are the effect that shear has when interfaces of varying softness are present or when applied to phases with positional order. Examples of attractive colloids where shear alignment is interesting for industrial applications are carbon nanotubes [10], wormlike micelles [11], or polymers.

In this Rapid Communication, we investigate by means of computer simulations the effect of large attractions on the I-N coexistence of systems of rods with aspect ratio 20. At rest, increasing the attraction transforms the nematic phase into the smectic and linearly decreases the interface width. In the presence of shear flow, a qualitatively new type of collective motion appears in which the director rotates in the

*m.ripoll@fz-juelich.de vorticity-flow plane. We infer that this rotation is related to the interaction of the rotating rods with the strong interface, such that it can be expected in other systems like dense colloidal rods suspensions in the proximity of a wall. This effect can therefore be of high relevance for microfluidic applications.

A hybrid simulation approach is employed. It combines molecular dynamics simulations for rodlike colloids with the mesoscale multiparticle collision dynamics (MPC) technique for the solvent [12]. Each rod is composed of $N_{m}=20$ monomers connected by harmonic springs that keep the distance $l$ between monomers constant and by bending potentials that provide rigidity to the rods such that the mean square endto-end distance is $0.98\left(N_{m}-1\right)^{2} l^{2}$ [13]. Interactions between rods are taken into account by the Lennard-Jones potential of attractive strength $\epsilon$ and monomer diameter $\sigma$. The repulsive part of the potential prevents that different rods interpenetrate. The attraction part mimics interaction between rods, as can be due to the presence of different smaller depleting components (e.g., polymers) diluted in the solvent. MPC consists of two alternating steps. In the streaming step, the solvent particles of mass $m$ move ballistically for a time $h$. In the collision step, the particles are sorted into the cells of a cubic lattice of cell size $a$. Particles in the same cell interchange momentum, such that the cell linear momentum and kinetic energy is conserved before and after the collision, ensuring that hydrodynamic interactions are properly taken into account; for details see Ref. [13].

The I-N phase coexistence can be found in a small window of packing fractions that depends on the rod aspect ratio, as predicted by Onsager theory [14] and confirmed by previous simulation results for rods with repulsive interactions [15]. Here, rods with $l=\sigma=0.25 a$ are considered, yielding a rod length $L \approx 5 a$. We include $N_{p}=10^{4}$ rods in a box of size $\left(L_{x}, L_{y}, L_{z}\right)=(4.4 L, 6 L, 4 L)$. This leads to a volume fraction of $\phi_{0}=0.125$, which is known to display I-N coexistence for rods of aspect ratio 20 . A purely nematic state is used as starting configuration, and all the rods are oriented in the same direction $(x)$, but their centers of mass display fluidlike order. After equilibration, part of the system has melted into an isotropic phase that coexists with an orientationally ordered phase. Configurations with large attraction strengths $\left(\epsilon>2.5 k_{B} T\right)$ are obtained starting from phase-separated configurations of a slightly lower $\epsilon$.

Large attractions induce additional positional order in the nematic, resulting in a smectic phase. The snapshot in Fig. 1 shows that the suspension has separated into two phases. In the isotropic phase, rods are oriented in all directions with 

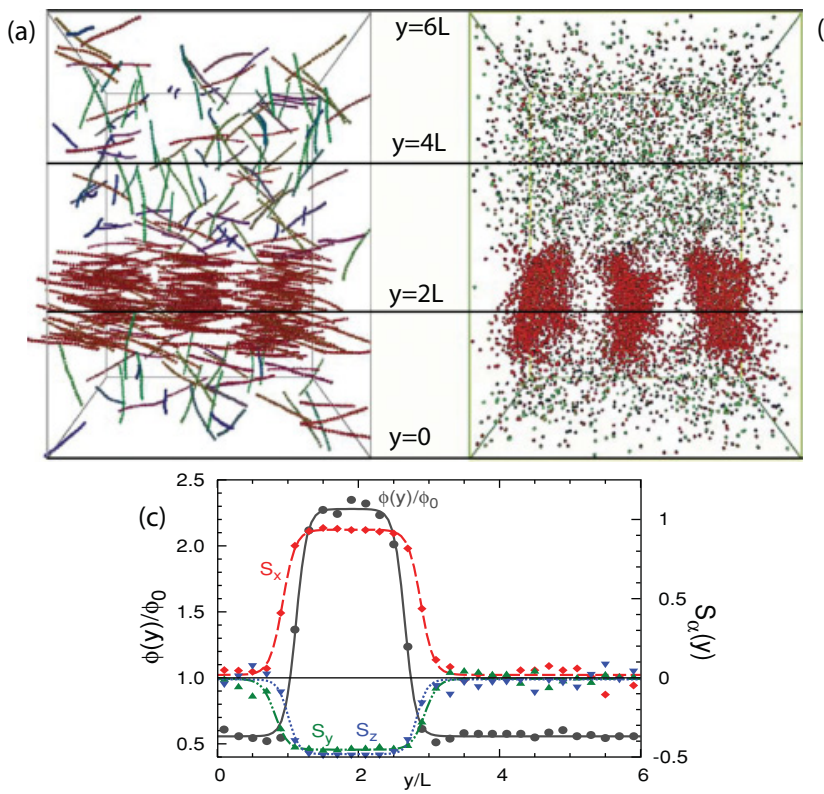

FIG. 1. (Color) Snapshot showing I-SmA coexistence with $\epsilon=$ $4.0 k_{B} T$. In (a) $4 \%$ of the rods are displayed. In (b) only a central bead of each rod is displayed. The orientation is color coded as follows: red is left-right, green is up-down, and blue is out-of-plane. (c) Density and orientational order parameter profiles, symbols show averages in planes $\perp y$, and lines show fits with Eq. (1).

equal probability, while in the ordered phase rods are mostly aligned parallel to the interface and grouped in three distinct layers. The rod's center-of-mass dispersion within each layer determines its width. Both this layer width and the number of layers are related to the system size and to the rods elasticity [16]. Thus, a slight increase of the box size in the horizontal direction may allow for a fourth layer with a decrease of the rod's center-of-mass dispersion. Furthermore, diffusion of rods from the isotropic phase through the smectic phase can be inferred given the eventual presence of rods in between the smectic layers and perpendicular to them.

The center-of-mass positions and the orientations of the rods are averaged in 30 slices along the gradient direction $y$. The orientational order parameter with respect to a direction $\mathbf{u}_{\alpha}$ in space is defined as $S_{\alpha}=\frac{1}{2}\left\langle 3 \cos ^{2} \theta-1\right\rangle$, where $\theta$ is the angle between the rod axes and the direction $\mathbf{u}_{\alpha}$. In the isotropic phase, the density $\phi$ is lower than the average value $\phi_{0}$, and the three $S_{\alpha}$ fluctuate around zero, as can be seen in Fig. 1(c). In the ordered phase instead, the density is larger than $\phi_{0}$ and differentiated values of the order parameter are obtained. The lack of order within the layers allows us to identify this phase as smectic-A (SmA). Density and order parameter profiles along the gradient direction [like those in Fig. 1(c)] are fitted to a double hyperbolic tangential function,

$$
\begin{aligned}
f(y)= & a\left\{\tanh \left[y-y_{0}-y_{1} / w\right]\right. \\
& \left.+\tanh \left[y-y_{0}+y_{1} / w\right]\right\}+d,
\end{aligned}
$$

where $w$ is the interface width and $2 y_{1}$ and $y_{0}$ are the width and the central position of the ordered phase. The density/order parameter in the isotropic phase is determined by $d$ and in the ordered phase by $d+2 a$. The accuracy of the

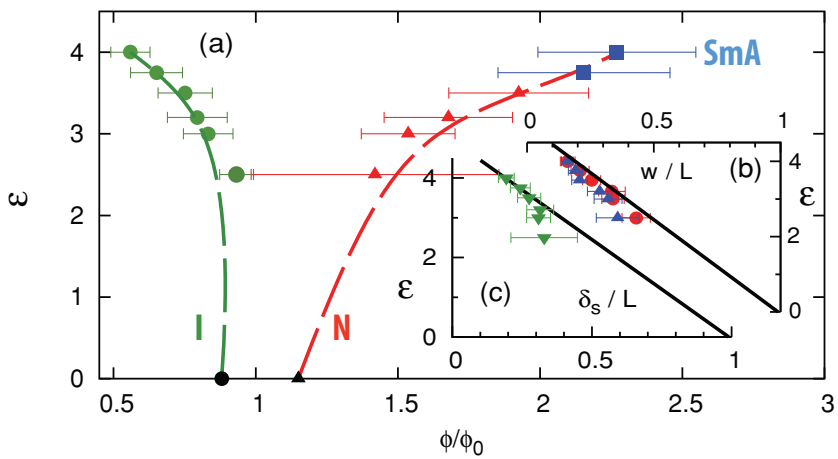

FIG. 2. (Color online) (a) Phase diagram of attractive colloidal rods as a function of the interaction strength $\epsilon$. The packing fraction $\phi$ is normalized to its average value $\phi_{0}$. Bullets correspond to the isotropic phase, triangles to the nematic, and squares to the smectic. Dashed lines are a guide to eye. (b) Width of the interface in units of rod lengths as measured from the density (triangles) and the orientational parameter (bullets) as a function of $\epsilon$. (c) Displacement of the density profile.

measurements is improved by averaging over the profiles of many configurations.

The phase diagram in Fig. 2(a) shows the density in the coexisting isotropic and ordered phases as a function of the interaction strength $\epsilon$. Values at vanishing attraction correspond to the Onsager limit [14]. Simulations show that the density difference of the coexisting isotropic and ordered phases increases weakly with $\epsilon$ for $\epsilon \lesssim 3 k_{B} T$, while it strongly increases for larger interaction strengths. In the ordered phase, the densities show a continuous change with the increase of the interaction strength, although around $\epsilon \simeq$ $3.75 k_{B} T$ the nematic becomes smectic A. This dependence qualitatively agrees with the behavior experimentally observed in suspensions of $f d$ viruses with dextran as a depletion agent [3].

The properties of the isotropic-nematic interface have been extensively studied in absence of attraction [17-19]. Here the interface width, $w$, is determined by fitting the density and the ordered parameter profiles to Eq. (1). Values obtained from the two profiles lead to very similar results and are shown in Fig. 2(b). The interface width, w, decreases linearly with increasing attraction. This is due to the increasing order with attraction in the nematic phase, which implies that the rods have less orientational freedom and the interface width shrinks. On the other hand, due to the effect of capillary waves [18,19], the absolute value of $w$ is strongly size dependent. We expect, though, that the decrease rate of $w$ with attraction will be size independent.

The orientational order parameter profile attributes to the nematic phase a larger thickness than the density profile, as can be seen in Fig. 1. This means that the order decays once the density has already been reduced to the isotropic value [17]. This effect is characterized by $\delta_{s}$, the interface displacement of the density profile. In Fig. 2(c), $\delta_{s}$ is displayed. Interestingly, $\delta_{s}$ has very similar values to $w$, also decaying linearly with $\epsilon$. Noticeably, there is no sign of the N-to-SmA transition in any of the previous quantities. Namely $w, \delta_{s}$, and the density of the the coexisting I-SmA phases vary continuously with $\epsilon$. 


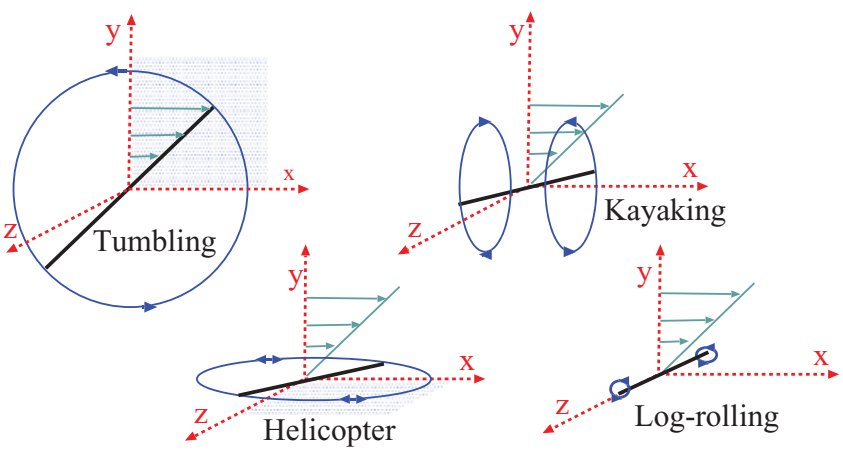

FIG. 3. (Color online) Illustration of the different rotation types of a rodlike colloid in shear flow: tumbling in the flow-gradient plane; kayaking tilted in the vorticity direction; helicopter in the flow-vorticity plane; and log-rolling where the rod lies in the vorticity direction and rotates around its axis.

Shear flow is imposed in the direction parallel to the interface. Lees-Edwards boundary conditions are employed, which for a homogeneous phase result in a linear velocity profile $\left(v_{x}, v_{y}, v_{z}\right)=(\dot{\gamma} y, 0,0)$, with $y$ the position in the gradient direction and $\dot{\gamma}$ the shear rate expressed in simulation time units $\sqrt{m a^{2} / k_{B} T}$. Order is induced by shear on the rods of the isotropic phase originating free space around each rod. Rods diffuse, then, from the large- to the small-density region [19]. For shear rates larger than a characteristic value, the system becomes homogeneous. For smaller shear rates, differences between phases remain, which determines the nonequilibrium phase diagram [7]. Furthermore, shear induces a collective rotation of the denser phase that coexists with a paranematic flow-aligned and less dense phase. Dispersions with small attractions rotate with tumbling or kayaking, this is with inclination in the gradient direction. Here we find a phenomenologically different behavior for rod dispersions

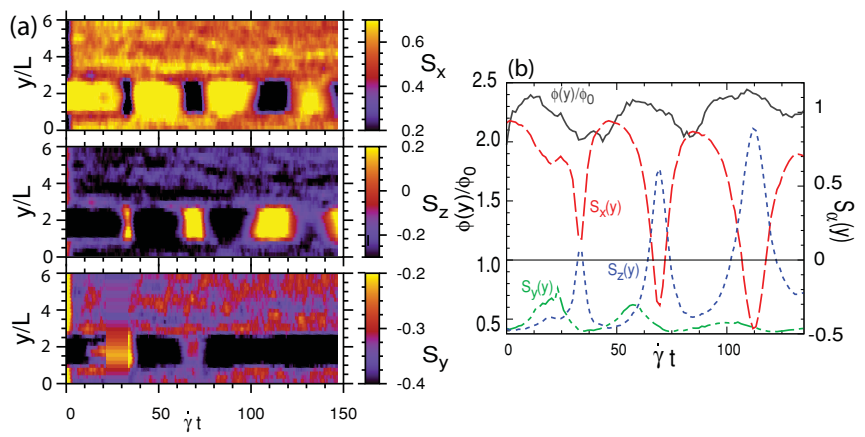

FIG. 5. (Color) Time evolution of $S_{\alpha}$. (a) Color code of values along the gradient direction. (b) Averages in the denser phase. The state corresponds to $\epsilon=4.0 k_{B} T$ and $\dot{\gamma}=0.005$.

with large attractions. Similar to the blades of a helicopter, the rotation plane is completely in the flow-vorticity plane. We refer to this as the helicopter rotation. Note that this rotation is essentially different from the so-called log-rolling [8], where each rod is permanently oriented in the vorticity direction and rotates around its main axis (Fig. 3).

Figure 4(a) shows a snapshot of a phase-separated system with a flow-oriented paranematic and a nematic phase with the director oriented in the vorticity direction, while undergoing a helicopter rotation. In this snapshot, an additional rotating cluster inside the paranematic-flow-aligned phase can be also observed. In Fig. 4(b) the density and order parameter along the gradient direction are analyzed. The presence of two intervals with larger density and order parameter in the vorticity direction can be quantitatively characterized. Figure 5 displays the time evolution of the normalized density $\phi / \phi_{0}$ and the orientational order parameter $S_{\alpha}$ in the three main axes. Starting from an unsheared configuration, the isotropic phase quickly orients with the flow while the ordered phase remains nematic. The first rotation may occur completely in the

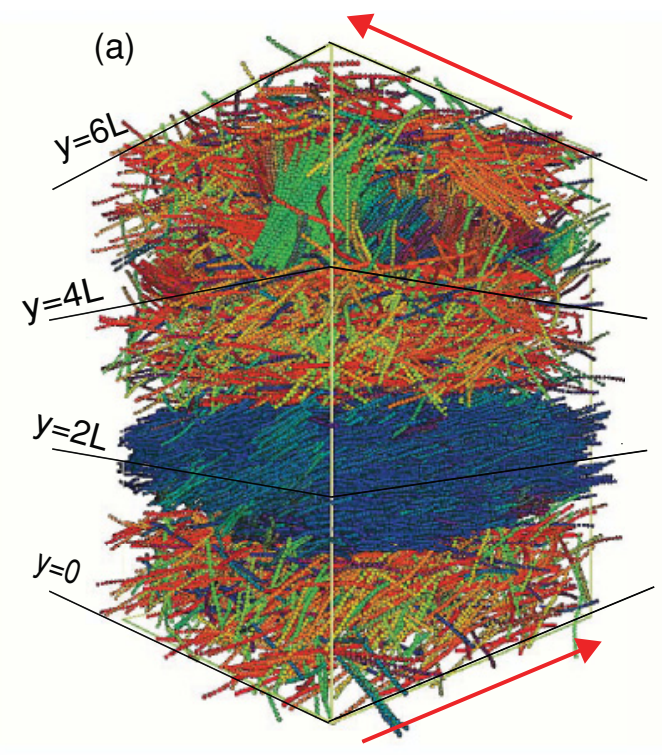

(b)

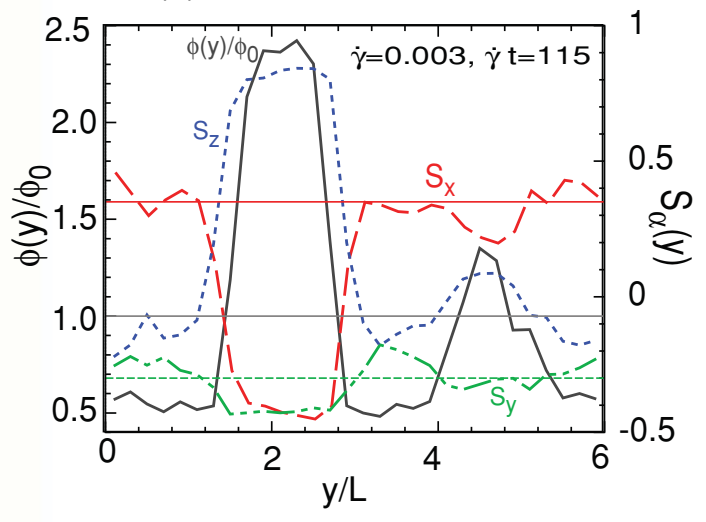

FIG. 4. (Color) (a) Snapshot of a state with a flow-oriented paranematic phase and a nematic phase undergoing a helicopter rotation. The state corresponds to $\epsilon=4.0 k_{B} T$ and $\dot{\gamma}=0.003$. Red arrows indicate the flow direction. (b) Averaged normalized density $\phi / \phi_{0}$ and orientational order $S_{\alpha}$ profiles of (a). Color codes similar to Fig. 1. For a movie, see Ref. [20]. 
flow-gradient plane, and then the rotation axis progressively tilts to the vorticity direction. The orientation in the gradient direction decreases to vanishing values, becoming a purely helicopter type of rotation. This can be seen for $\dot{\gamma}=0.005$ in Fig. 5 and for other shear rates in Ref. [20].

In the flow-vorticity plane, none of the two directions is preferred such that spontaneous symmetry breaking is required to perform the helicopter rotation. As a result, the rotation occurs in both directions. Some rotation cycles are completed $\left(180^{\circ}\right)$, and some change direction before such that the appearance of rheochaos (chaotic stress-strain curves and/or orientational dynamics [21]) is to be expected. Incomplete rotations in the flow-gradient plane are known as wagging such that we could call this behavior helicopter-wagging.

Simulations are initiated from steady configurations with I-SmA phase coexistence with $\epsilon=4.0 k_{B} T$, or with I-N coexistence corresponding to a lower $\epsilon$, case in which attraction and shear flow are increased at the same time. No significant differences are found. The sequence tumblingkayaking-helicopter is followed in all investigated cases. The smectic layers, initially perpendicular to the interface, become tilted, reducing their distance. The rod diffusion among layers is then enhanced such that the positional order is progressively deconstructed (more details in Ref. [20]). The SmA phase becomes, then, nematic under shear, in contrast to what has been found in systems with homeotropic alignment or intrinsic layering [22]. Although the dynamic transition to the helicopter rotation seems to take place at a similar interaction strength as the appearance of I-SmA in equilibrium, we find no direct connection between the two features.

A possible explanation for the transition from tumbling into helicopter rotation is the following. During the rotation in the flow-gradient plane, the anchoring angle of the orientation of the dense phase at the interface is time dependent. Configurations with the nematic director perpendicular to the interface are energetically much more unfavorable than configurations with the rods aligned along the flow such that they occur during briefer times. The related surface tension has not been measured in this work, but it is sensible to think that it noticeably increases with attraction. From a certain $\epsilon$, it probably becomes too large, making the rotation in the flow-vorticity plane more efficient. As a consequence, this helicopter rotation might be expected as well for colloidal rods systems in the proximity of a wall. It can also be speculated that for confined dense systems, clusters of rods undergoing helicopter oscillations close to a wall would separate from each other in regions perpendicular to the wall, which could be related to the still unclear phenomena of vorticity-band formation [23].

I want to thank M. P. Lettinga, G. Gompper, R. G. Winkler, M. P. Allen, G. A. Vliegenthart, and R. L. C. Vink for helpful discussions. Generous allocation of computer time is acknowledged to to the John von Neumann Institute for Computing (NIC) at the Research Centre Jülich.
[1] P. G. De Gennes and J. Prost, The Physics of Liquid Crystals (Oxford University Press, Oxford, UK, 1993).

[2] P. G. Bolhuis et al., J. Chem. Phys. 107, 1551 (1997).

[3] Z. Dogic, Phys. Rev. Lett. 91, 165701 (2003); Z. Dogic, K. R. Purdy, E. Grelet, M. Adams, and S. Fraden, Phys. Rev. E 69, 051702 (2004).

[4] T. Schilling and D. Frenkel, Nature 332, 822 (1988); Phys. Rev. Lett. 92, 085505 (2004); G. Cinacchi and L. De Gaetani, Phys. Rev. E 77, 051705 (2008); A. Cuetos et al., Faraday Discuss. 144, 253 (2010).

[5] G. R. Luckhurst et al., Liq. Cryst. 8, 451 (1990); E. de Miguel et al., Mol. Phys. 74, 405 (1991).

[6] M. A. Bates, Chem. Phys. Lett. 288, 209 (1998).

[7] M. Ripoll et al., Phys. Rev. Lett. 101, 168302 (2008); J. Phys. Condens. Matter 20, 404209 (2008).

[8] R. G. Larson and H. C. Öttinger, Macromolecules 24, 6270 (1991); P. D. Olmsted and C. Y. David Lu, Phys. Rev. E 60, 4397 (1999).

[9] Y. G. Tao, W. K. denOtter, and W. J. Briels, Rheol. Acta 42, 20 (2003); Y.-G. Tao et al., Phys. Rev. Lett. 95, 237802 (2005).

[10] E. K. Hobbie and D. J. Fry, Phys. Rev. Lett. 97, 036101 (2006); E. K. Hobbie, Rheol. Acta 49, 323 (2010).

[11] G. Petekidis et al., Langmuir 18, 4248 (2002); C. Barentin and A. J. Liu, Europhys. Lett. 55, 432 (2001).

[12] A. Malevanets and R. Kapral, J. Chem. Phys. 110, 8605 (1999); T. Ihle and D. M. Kroll, Phys. Rev. E 63, 020201(R) (2001).

[13] R. G. Winkler et al., J. Phys. Condens. Matter 16, S3941 (2004); M. Ripoll et al., Europhys. Lett. 68, 106 (2004); M. Ripoll,
K. Mussawisade, R. G. Winkler, and G. Gompper, Phys. Rev. E 72, 016701 (2005).

[14] L. Onsager, Ann. N.Y. Acad. Sci. 51, 627 (1949).

[15] H. Fynewever and A. Yethiraj, J. Chem. Phys. 108, 1636 (1997).

[16] P. van der Schoot, J. Phys. II (France) 6, 1557 (1996).

[17] M. P. Allen, J. Chem. Phys. 112, 5447 (2000); M. S. Al-Barwani and M. P. Allen, Phys. Rev. E 62, 6706 (2000); R. L. C. Vink and T. Schilling, ibid. 71, 051716 (2005); R. L. C. Vink et al., J. Chem. Phys. 123, 074901 (2005); J. Elgeti and F. Schmid, Eur. Phys. J. E 18, 407 (2005).

[18] N. Akino, F. Schmid, and M. P. Allen, Phys. Rev. E 63, 041706 (2001); S. Wolfsheimer, C. Tanase, K. Shundyak, R. vanRoij, and T. Schilling, ibid. 73, 061703 (2006).

[19] G. Germano and F. Schmid, J. Chem. Phys. 123, 214703 (2005).

[20] See supplemental material at [http://link.aps.org/supplemental/ 10.1103/PhysRevE.83.040701].

[21] S. H. L. Klapp and S. Hess, Phys. Rev. E 81, 051711 (2010); G. Rienäcker, M. Kroger, and S. Hess, ibid. 66, 040702(R) (2002); G. Rienäcker et al., Physica A 315, 537 (2002).

[22] T. Soddemann et al., Eur. Phys. J. E 13, 141 (2004); D. Kramer and H. Finkelmann, Phys. Rev. E 78, 021704 (2008); O. Stenull, T. Lubensky, J. M. Adams, and M. Warner, ibid. 78, 021705 (2008); S. Koschoreck et al., Rheol. Acta 48, 231 (2009).

[23] J. K. G. Dhont and W. J. Briels, Rheol. Acta 47, 257 (2008); K. Kang, M. P. Lettinga, Z. Dogic, and J. K. G. Dhont, Phys. Rev. E 74, 026307 (2006); K. Kang et al., Rheol. Acta 47, 499 (2008). 